\title{
A NEW OCULAR MICROMETER FOR THE MAHIS
}

\author{
T. R. Kirian, \\ Pulkovo Observatory \\ V. S. Korepanov and \\ Institute of Precise Mechanics and Optics \\ V. M. Grozdilov \\ Pulkovo Observatory
}

A working model of an ocular micrometer has been designed for the MAHIS (Meridian Automated Horizontal Instrument by Sukcharev). An optical scheme of the micrometer includes the following devices:

a) Artificial light marks in the focal plane of the objective. The marks have an increased sharpness and have a stable scale factor under defocusing. An amplitude-phase grid illuminated by coherent light from the second main point of the objective is used to make the marks.

b) The observed stars and artificial marks are imaged on the CCD chip by means of an additional objective. This objective also corrects chromatic aberations of the main objective.

c) A concentric meniscus is used to compensate for the chromatic refraction of the atmosphere. The meniscus center of curvature coincides with the center of the system's pupil image. In this case the compensation is equal at all points of the field of view.

d) The possibility of measuring the normal attitude of the flat mirror relative to the main instrumental plane during each observation is discussed. For these purposes there needs to be a holographic grid on the mirror surface, an artificial zenith or nadir horizon and an autocollimated source of light in the focal plane.

e) Laboratory investigations of a working model of a CCD camera are being carried out and the basic software is being developed. This work is planned to be finished in the autumn of 1994. The CCD matrix ISD 011A (NPO "Electron", St. Petersburg, Russia) 512 x 512 pixels, with pixel size of $16 \times 16 \mathrm{mkm}$ is used. The noise is 5 electrons/pixel/sec when the temperature is $-40^{\circ} \mathrm{C}$ (thermoelectric cooler).

The basic CCD software is being adjusted in the following way. First the CCD control and information processing algorithms are to be debugged with help of an IBM PC (the software is written in the $\mathrm{C}$ programming language with the use of floating point codes). Then, after a real CCD image has been obtained, the algorithms are optimized for the specialized 
signal processor TMS320C3x (also with the use of a C-compiler). Then, if necessary, they are transformed into fixed-point TMS' codes. The DSR-adapter used with its TMS320C31 processor and with the 8-Mbyte RAM allows real-time work for the two chosen modes of the micrometer. The first one is the momentary readings of the CCD frames and the second one is so called the "time delay and shifts" mode (the TMS320C25 signal processor allows only the fixed frame mode). More detailed information concerning the new MAHIS CCD camera will be available after tests and refinements of the CCD software. 